\title{
Packet Equalisation in PONs using Adjustable Gain-Clamped Semiconductor Optical Amplifiers (AGC-SOA)
}

\author{
Lin Liu, Craig Michie, Anthony E. Kelly*, Ivan Andonovic \\ Department of Electronic and Electrical Engineering, University of Strathclyde, Glasgow G1 1XW, Scotland \\ * Department of Electronics and Electrical Engineering, University of Glasgow, Glasgow G12 8LT, Scotland \\ Tel: (+44) 141548 2521, Fax: (+44) 141552 4968, e-mail: c.michie@eee.strath.ac.uk
}

\begin{abstract}
The past ten years have witnessed a dramatic growth in the delivery of high bandwidth services through Passive Optical Networks (PONs), directly to the home or to the kerb. The bursty nature of upstream traffic in a PON (from the user to Optical Network Unit, ONU) combined with a wide dynamic range of signal strength $(-15 \mathrm{~dB}$ to $-28 \mathrm{~dB}$ the difference between a very close Optical Network Terminal (ONT) with a small split ratio and a distant ONT with a high split ratio), places severe demands on the burst mode receiver at the ONU. We report here on an adjustable gain-clamped semiconductor optical amplifier (AGC-SOA) designed to maximize the output saturated power while adjusting gain to regulate the power differences between packets without loss of linearity. This device is shown to be able to modulate gain at rates that are compatible with packet to packet equalisation. Through theoretical analysis coupled with experimental verification, this paper demonstrates for the first time that this is entirely feasible.
\end{abstract}

Keywords: Semiconductor optical amplifier, Psat, gain modulation.

\section{INTRODUCTION}

The growing penetration of home DSL connections demonstrates clearly that demand for high bandwidth services exists. Realistic future service scenarios predict traffic growth in excess of 50\% per year [1]. Analysis shows that to sustain this level of growth economically requires a significant reduction in the amount of equipment (interfaces between nodes) in the network. These drivers have thus stimulated interest in access solutions based upon Passive Optical Networks (PONs) with high (1024) splitting ratios [1]-[2]. Central to this, in particular for extended reach PONs which may cover distances of $100 \mathrm{~km}$ or more [3], is the need for low cost optical amplifier technologies. Semiconductor optical amplifiers (SOAs) have a clear role to play in this context. The wavelength plan for future $10 \mathrm{Gbit} / \mathrm{s}$ (XG-PON) [2] uses wavelengths from $1260 \mathrm{~nm}$ up to $1620 \mathrm{~nm}$ and alternative optical amplifier technologies do not function efficiently across this band.

Upstream traffic in a PON (from the user to the Optical Network Unit, ONU) is normally time division multiplexed (TDM) with a wide variation in path loss arising from differences in transmission distances and splitting losses. The bursty nature of this traffic combined with a wide dynamic range of signal strength $(-15 \mathrm{~dB}$ to $-28 \mathrm{~dB}$ - the difference between a very close Optical Network Terminal (ONT) with a small split ratio and a distant ONT with a high split ratio), places severe demands on the burst mode receiver at the ONU. In particular, the design of trans-impedance and limiting amplifiers and avalanche photo diodes is complicated by the need to cater for this wide dynamic range. As the upstream data rate increases, and longer reach PONs are deployed, this issue will become even more critical. The dynamic adjustment of the gain of optical amplifiers in order to regulate path losses and the signal strength at a packet level could substantially alleviate many of the above issues in long reach PONs. Signal strengths will be presented at the ONU at an optimized power level with concomitant advantages in reducing the receiver dynamic range requirement thus reducing its overall cost. To be of value, the timescale of the gain adjustment should take place at least within the guard band of the packet/frame transmission. For 10G PON systems this implies that the amplifier be able to regulate its gain, without loss of Psat, within a $26 \mathrm{~ns}$ timescale, (64 bits at $2.5 \mathrm{Gbit} / \mathrm{s}$ on the upstream direction) [4]. Potentially, the dynamic control of the optical amplifier can be implemented through the standard GPON protocol which provides knowledge of distance to a transmitting node. Alternatively, a rapid control circuit based on high speed measurements of signal strength may be appropriate.

We report here on an adjustable gain-clamped semiconductor optical amplifier (AGC-SOA) designed to maximize the output saturated power while adjusting gain to regulate the power differences between packets without loss of linearity. Fig. 1a illustrates conceptually, the design of the AGC-SOA which has been reported in [5]. The architecture comprises two active (amplifier) regions defining a data path through the signal SOA (SOA1) and a laser cavity containing SOA1 and a control SOA (SOA2). SOA1 amplifies light in the signal path. The lasing mode derives gain from both SOA1 and SOA2. The composite gain provided by both SOAs which in combination, regulates the condition for the onset of lasing and hence the carrier concentration (gain) of the signal SOA. Hence by controlling the drive to SOA2, the gain imparted by SOA1 can be adjusted. SOA1 is continually operated at full current, and therefore the AGC-SOA allows signals to be amplified by SOA1 at a clamped gain varied by SOA2, maximising the saturation output power and thereby maintaining an extended linear regime [5]. The main advantage of the proposed approach is that there are no mechanical tuning elements 
and hence the gain can be adjusted via direct electrical control potentially at ns timescales. While the operation of this device has been presented previously for the static gain case [5], it's behaviour under dynamic gain modulation conditions is not well understood. Here we report on some theoretical modelling that we have undertaken, supported by experimental analysis, which suggests that gain adjustment and stabilisation within the timeframe required is possible.

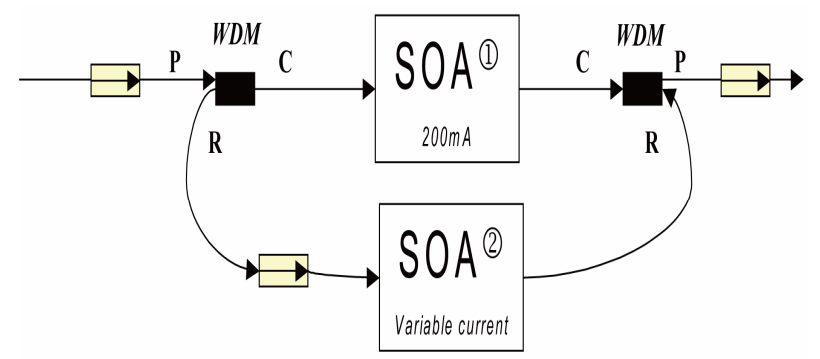

a)

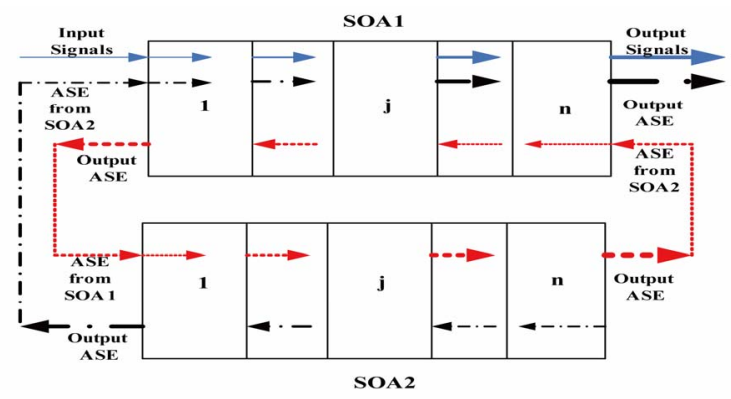

b)

Figure 1. Counter propagating ring laser AGC-SOA: a) experimental configuration, b) simulation model.

\section{MODEL OF AGC-SOA}

The numerical model [6] for the whole system of Fig. 1b is achieved using iterative circulations. In the first iteration, the amplified spontaneous emission (ASE) in both directions of SOA2 is calculated assuming no ASE power is coupled in. Then the ASE generated by SOA2 travels in both clockwise and counter-clockwise order towards SOA1. Under this boundary condition, the ASE originated from SOA2 together with the one generated by SOA1 is amplified by SOA1 as it travels through, however only the backward ASE inside the SOA1 is sent to SOA2. For any successive iterations, the ASE from SOA1 couples into SOA2 before SOA2 generates ASE. When ASE travels inside the ring cavity, the facet reflectivity and coupling loss of both SOAs, the insertion loss for the isolator and WDM couples are taken into account. The round trip time is around 1.67ns viz. the fibre length is about $0.5 \mathrm{~m}$ (according to new experimental setup). Therefore, fibre loss and dispersion are neglected in the simulation. The iterative procedure is terminated when the maximum difference of the ASE powers at each discrete frequency band between successive iterations is less than the desired tolerance. The whole numerical model is implemented using Matlab.

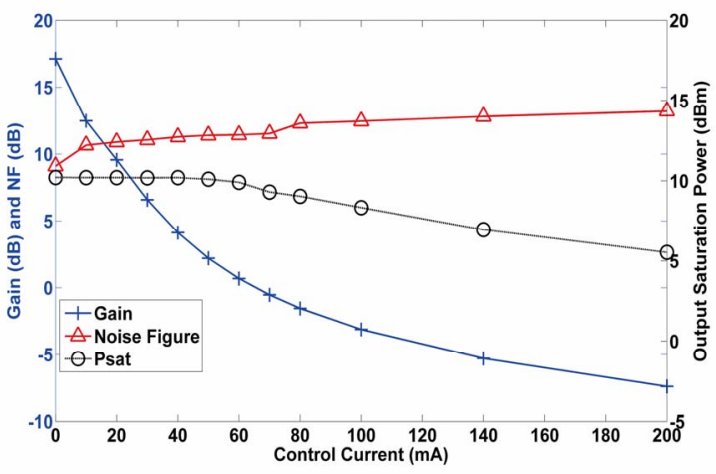

Figure 2. Modeled steady state AGC-SOA performance.

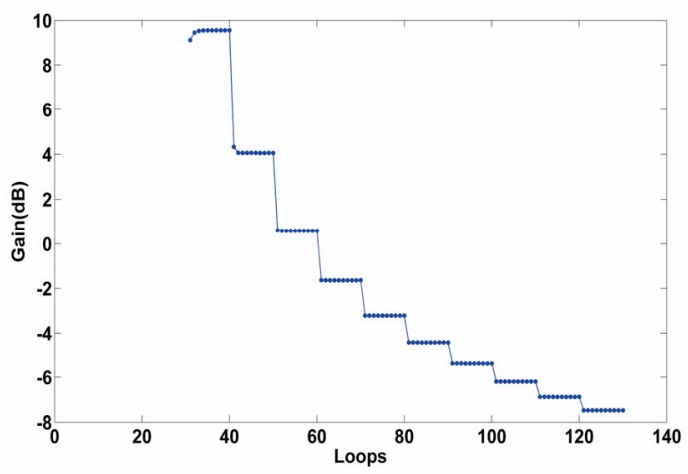

Figure 3. Dynamic gain variation with clamping current.

The DC parametric operation of the AGC-SOA can be estimated using the above model: a CW light (1550 nm) is introduced into AGC-SOA after it stabilised, the input signal power is steadily increasing from $-35 \mathrm{dBm}$ to $20 \mathrm{dBm}$, the normal performance metrics of Gain, maximum output power at gain saturation (Psat) and noise figure (NF) are then recorded under a given clamping current condition, the clamping current is change from $0 \mathrm{~mA}$ to $200 \mathrm{~mA}$. The simulation results are presented as a function of clamping current in Fig. 2 . The overall trends of these models are in reasonable agreement with previously reported experimental characterizations. Gain clamping begins at a clamping bias current of greater than $0 \mathrm{~mA}$. Experimentally this value was observed to be nearer $10 \mathrm{~mA}$ before here there was sufficient gain within the clamping SOA to overcome loop losses and allow the lasing mode to stabilize. This difference is mainly due to the overestimation of ASE noise in the model. Strong clamping is observed when the control SOA is operated at high gain levels. Here the AGC-SOA is driven into attenuation. As the gain is clamped, the NF can be seen to increase as the gain is clamped but in the main, the Psat value remains relatively constant when the clamping current is less than $100 \mathrm{~mA}$.

The main focus of the present work is to better understand the dynamic behaviour of the AGC-SOA. In order to do this, the model was run under the following conditions. Firstly stable operation of the AGC-SOA was 
ensured by running the simulation with only ASE for the first 30 loop iterations and with the SOA2 (clamping SOA) bias of $20 \mathrm{~mA}$. At this point a $0 \mathrm{dBm}$ input signal was introduced and it can be seen that the gain of the AGC-SOA is around $10 \mathrm{~dB}$. The clamping SOA bias current was then increased every 10 loops, from $20 \mathrm{~mA}$ to $200 \mathrm{~mA}$, in steps of $20 \mathrm{~mA}$ and the gain change observed as in Fig. 3. It is clear from these experiments that the gain is adjusted and stabilized within 1 or 2 loops of the model iteration. This implies therefore that the gain can be adjusted within nanosecond timescales which is what is required for dynamic packet equalisation.

\section{EXPERIMENTAL VERIFICATION}

The theoretical modelling of the AGC-SOA predicts that the gain can be modified within a timescale of nanoseconds. In a dynamic packet equalisation scenario, the AGC-SOA must be able to adjust and stabilise it's gain within the period of the guard band of the PON transmission, 32 bit periods - approximately $26 \mathrm{~ns}$. Similarly for $10 \mathrm{G}$ PON systems (64 bits at $2.5 \mathrm{Gbit} / \mathrm{s}$ on the upstream direction) equates to $26 \mathrm{~ns}$ [4]. The model therefore predicts that this is feasible.

Central to attaining high speed gain modulation is the capability to directly modulate the gain of the clamping SOA via its drive current. Reflective SOAs, packaged in a TO can, have been designed to both amplify and modulate signals at data rates in excess of $1 \mathrm{Gbit} / \mathrm{s}$ [7]. The topology of the AGC-SOA was therefore modified to include an RSOA as shown below in Fig. 4. Signals to be amplified are input through the P (pass) port on the WDM coupler. C-band signals are passed through to the SOA. At the output of the SOA, the amplified C-band signal is passed through the WDM coupler on the output. Out of band signals, ASE from the S-band and L-band signals are amplified by the RSOA and used to form a counter propagating clamping mode.

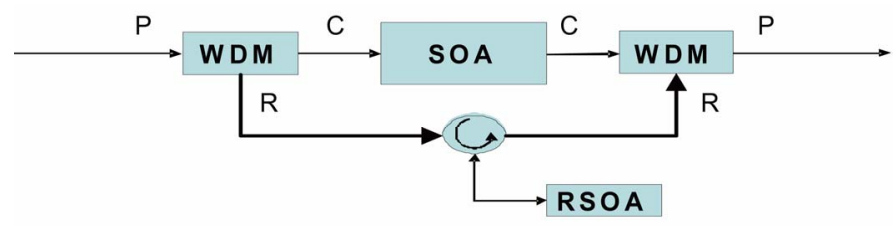

Figure 4. RSOA regulated AGC-SOA.

With the set up as shown in Fig. 4, the RSOA was driven with a data stream between two gain extremes, high gain and low gain. In the RSOA high gain state, the gain of the AGC-SOA is highly clamped, i.e. it has very low gain. Conversely, when the RSOA is turned to a low gain state, the gain of the AGC-SOA is high. The transition time between the two states represents the minimum time required to adjust and settle the gain of the AGC-SOA. Fig. 5 shows the variation in ASE signal emitted by the in line SOA in response to directly modulating the RSOA gain. The transition between a high and a low state is in response to the $\overline{d a t a}$ signal. This shows clearly that this transition time is less than 2 ns.

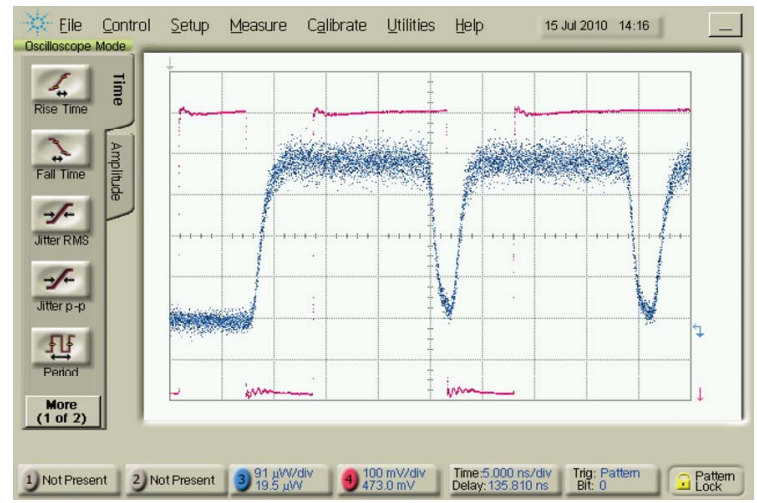

Figure 5. Directly modulated SOA ASE.

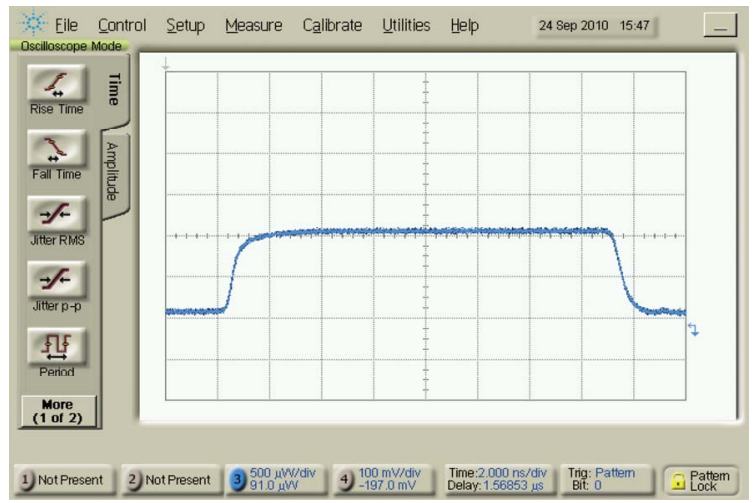

Figure 6. Directly modulated signal.

The experiment was repeated with a steady state signal of zero $\mathrm{dBm}$ presented at the input to the AGC-SOA, Fig. 6 shows that the amplitude of the seed signal has been directly modified through changing the gain of the AGCSOA. Again it is clear that the transitions between gain states are less than $2 \mathrm{~ns}$ ( $2 \mathrm{~ns}$ per division timescale). The experimental characterization and the modelling are therefore in broad agreement and indicate that the gain of the AGC-SOA can be adjusted and stabilized within the $26 \mathrm{~ns}$ timescale that would be required for packet equalisation in PONs.

High speed gain modulation of the AGC-SOA on a data signal was demonstrated using the experimental arrangement shown in Fig. 7. Data from an Agilent N4903A J-BERT Bit Error Rate test set was used to modulate a CWDM transmitter module (Finisar FDB 1027) at a data rate of $1.25 \mathrm{Gbit} / \mathrm{s}$. The signal amplitude 
was then regulated using a combination of an Erbium Doped Fibre Amplifier (EDFA) and a variable optical attenuator (VOA). The output of the VOA was then input to the AGC-SOA. The gain of the AGC-SOA was regulated by applying a square wave to the RSOA via an ETS3869 laser driver, which provides control over the bias current (Ibias) and the data modulation power. The output from the AGC-SOA was detected using a pin photoreceiver and displayed on an Agilent Digital Communications Analyzer (DCA). All instruments were synchronised from a common clock source so that modulation and data signals could be observed at the same time.

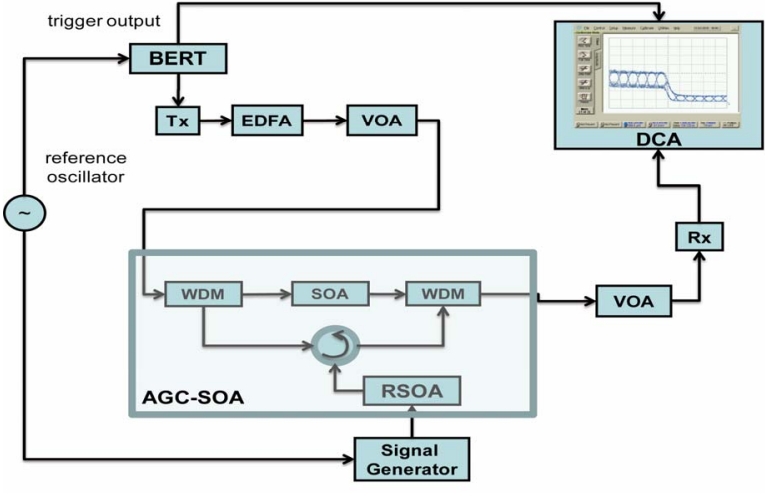

Figure 7. Gain equalisation experiment.

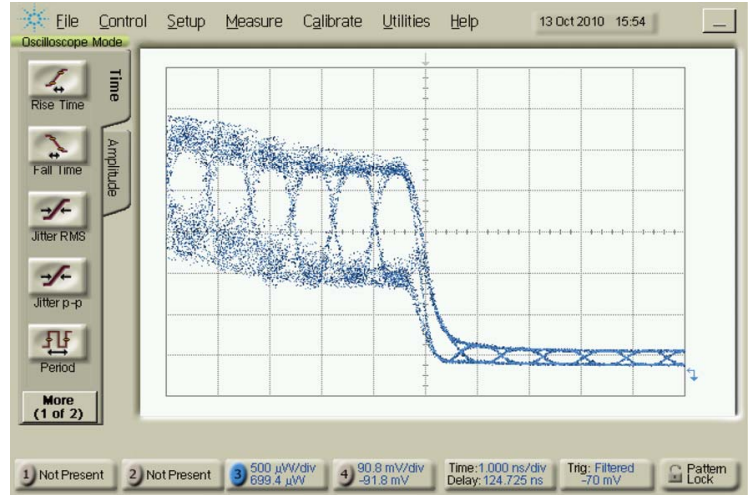

Figure 8. Eye diagram of gain modulated data signal.

An eye diagram of the recovered data signal is shown below in Fig. 8. It shows the output from the SOA presented with a signal level that is sufficiently high to produce a degree of patterning. The gain of the AGCSOA is then rapidly reduced to the point where the onward transmitted signal is much reduced but is substantially cleaner. The transition from the high gain state to the low gain state takes place over two bit periods, just over 2 ns.

\section{CONCLUSIONS}

This paper has presented a theoretical analysis of an AGC-SOA. Both the steady state operation and the case where the gain of the AGC-SOA is dynamically modulated are addressed. The dc parametric behaviour is shown to agree well with experimental measurements. An adaptation of the models has shown that in principle, the gain of the AGC-SOA can be regulated dynamically to respond within the guard band of a PON transmission. Gain settling times within the order of $2 \mathrm{~ns}$ are predicted. This analysis was shown to agree well with experimental evaluation of the AGC-SOA behaviour which demonstrated gain modulation and settling within tow bit periods of a $1.25 \mathrm{Gbit} / \mathrm{s}$ data signal. A key motive for this study was to better understand the dynamic behaviour of the AGC-SOA with a view to establishing a means for dynamically modulating the gain of the AGC-SOA in response to changes in packet amplitude. For this to be effective, the gain should be adjusted and stabilised within a timespan of less than $20 \mathrm{~ns}$. Both the theoretical analysis and the experimental investigation indicate that it is possible.

\section{ACKNOWLEDGEMENTS}

Strathclyde University gratefully acknowledges funds received from the British Council under the Prime Ministers Initiative II, Research Cooperation. This work was also supported in part by the Glasgow Research Partnership in Engineering (GRPe).

\section{REFERENCES}

[1] R. P. Davey , D. B. Payne: The future of optical transmission in access and metro networks - An operator's view, in Proc. ECOC 2005, Glasgow, U.K., September 2005, Vol. 5, pp. 53-56.

[2] F. J. Effenberger: The XG-PON system: cost effective 10Gb/s access, J. Lightw Technol., vol. 29, no. 4, pp. 403-409, Feb. 2011.

[3] R. P. Davey, P. Healey, I. Hope, P. Watkinson, D. B. Payne, O. Marmur, J. Ruhmann and Y. Zuiderveld: DWDM reach extension of a GPON to 135 km, J. Lightw Technol., vol. 24, no. 1, pp. 29-31, Jan. 2006.

[4] ITU-T Recommendation G.987.2: 10-Gigabit-capable passive optical networks (XG-PON): Physical media dependent (PMD) layer specification, Jan. 2010.

[5] C. Michie, A. E. Kelly, I. Armstrong, I. Andonovic and C. Tombling: An adjustable gain-clamped semiconductor optical amplifier (AGC-SOA), J. Lightw Technol., vol. 25, no. 6, pp. 1466-1473, Jun. 2007.

[6] L. Liu, C. Michie, A. E. Kelly, I. Andonovic: Numerical analysis of adjustable gain-clamped semiconductor optical amplifier (AGC-SOA) performance, in Proc. ICTON 2010, Munich, Germany, June 2010, paper MoP1.

[7] D. C. Kim, B. S. Choi, H. S. Kim, K. S. Kim, O. K. Kwon, and D. K. Oh: 2.5 Gbps operation of RSOA for low cost WDM-PON sources, in Proc. ECOC, 2009, Vienna, Austria, September 2009, pp. 1-2. 